\title{
cubierta de aparcamiento de acero desmontable en Eschborn República Federal Alemana
}

Klaus Idelberger, ingeniero 508-6

\section{sinopsis}

Se describe un aparcamiento de dos niveles, constituido por una estructura metálica, con forjados de hormigón armado a base de unidades de $3,5 \times 2,25 \times 0,15 \mathrm{~m}$, con enriñonado posterior.

La construcción es desmontable, con vistas a traslados o posibles ampliaciones, lo cual constituye una caracteristica funcional y diferencial.

En la actualidad se dispone de 68 plazas, distribuidas en una superficie de $24 \times 35$ metros.

El concepto del "edificio de aparcamiento" caracteriza unas superficies de aparcamiento para vehículos motorizados apiladas en varias plantas. Bajo la definición de "cubierta de aparcamiento" se entiende, como máximo, niveles de aparcamiento en número de dos, o en la mayoria de los casos sólo una planta, pensados por regla general para el aparcamiento de los turismos.

El edificio de aparcamiento de dos plantas - ampliable a diez- de la fábrica de productos farmacéuticos Ciba-Geigy, de Schweizerhalle, posee desde el año 1976 ocho plantas completas, con lo que ocupa el primer lugar en Europa.

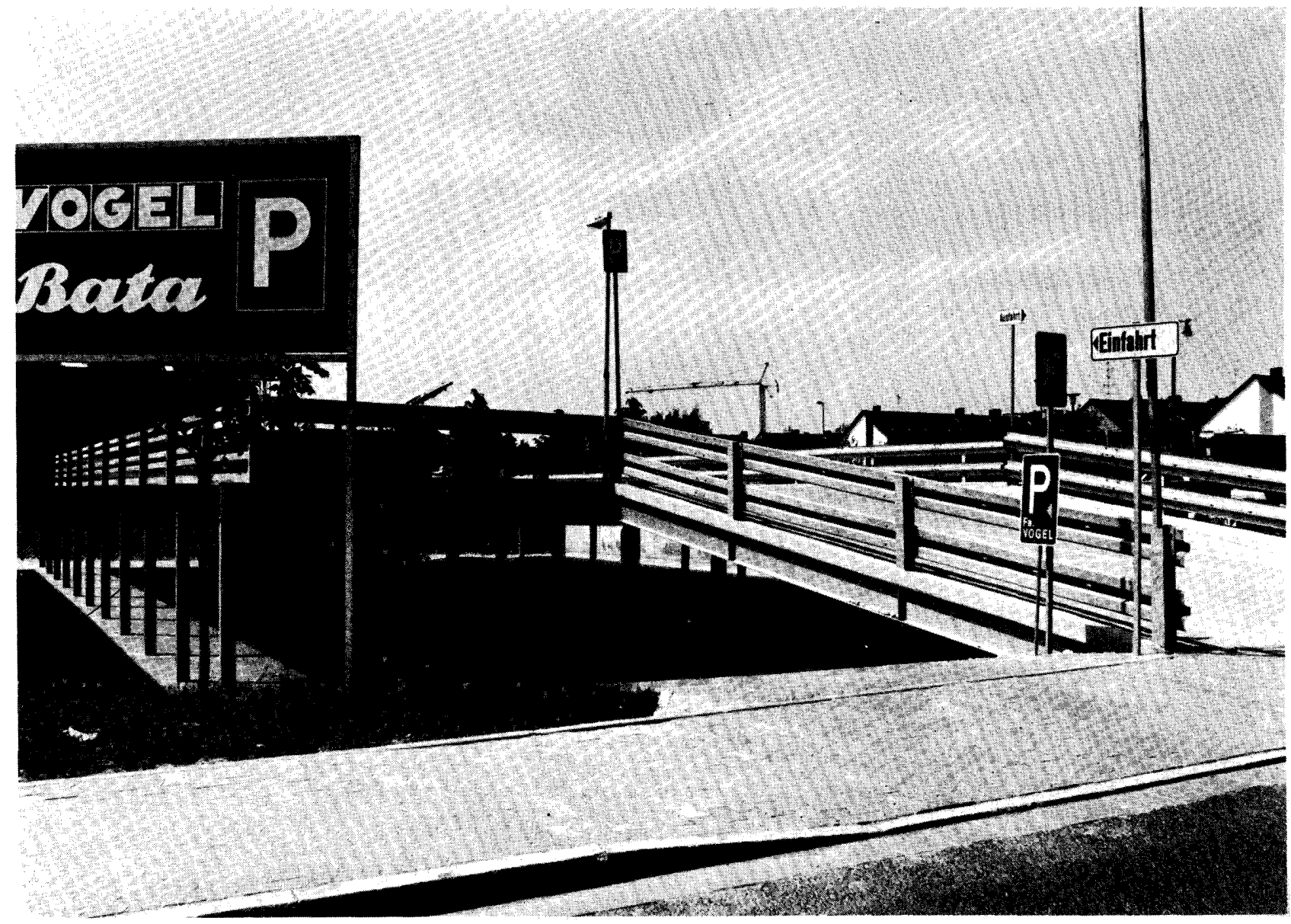




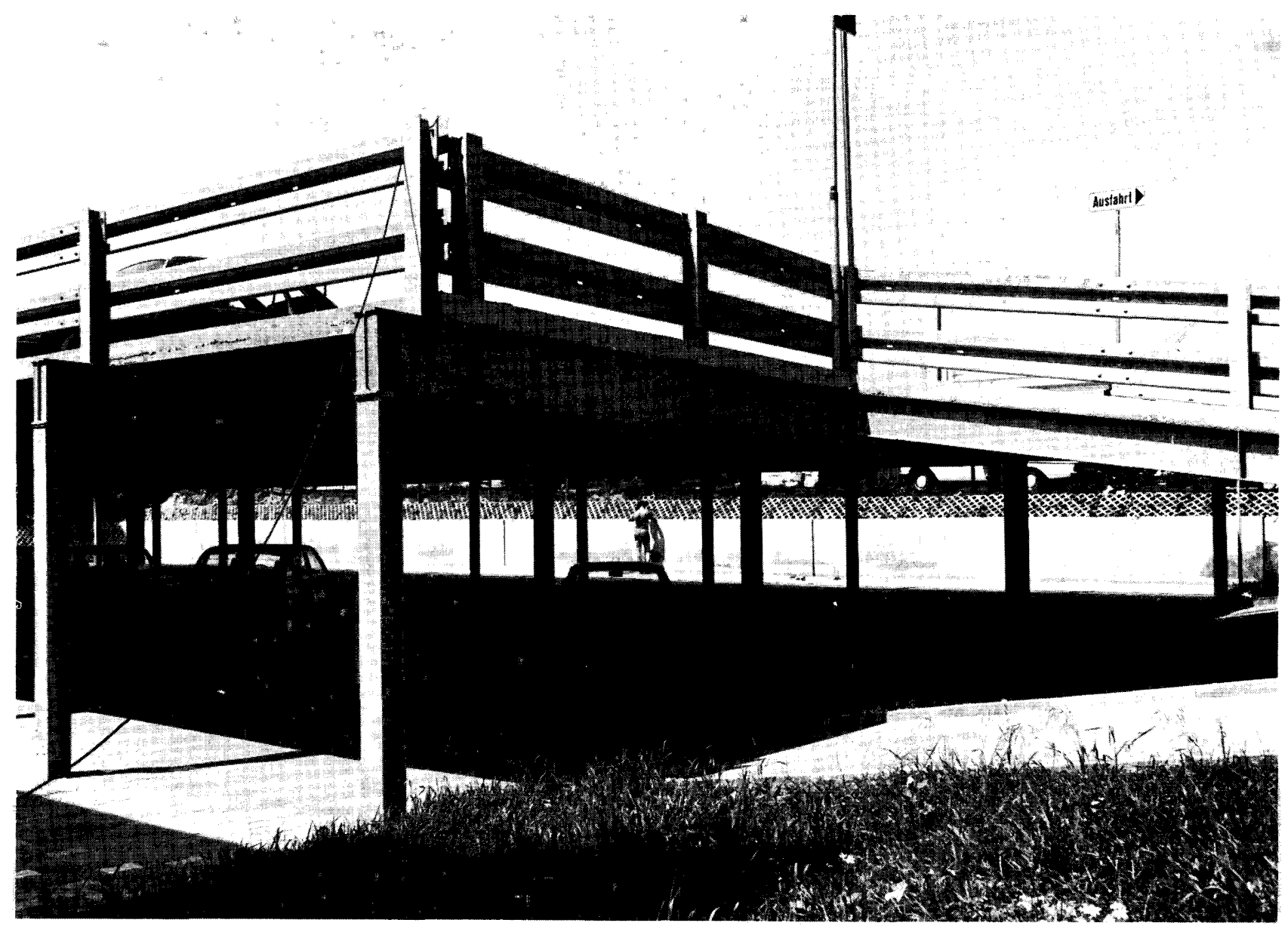

Ocho semiplantas superpuestas, correspondientes a cuatro plantas completas, tiene el edificio de aparcamiento 0 , mejor dicho, el edificio de exposición de automóviles Becker de Düsseldorf —con la última planta construida en acero- desde el mes de agosto de 1979. Ya en 1972 se amplió el aparcamiento de la BOAC/BAC, en el aeropuerto de Londres-Heathrow, de dos a tres plantas completas utilizándose un tipo de forjados sobre celosia espacial de acero. Por encima de la cubierta del aparcamiento existente en la Escuela Superior de Medicina de Hannover se construyó, en 1978, una cubierta de aterrizaje para helicópteros y un aparcamiento. Finalmente se duplicó también, prácticamente, el número de plazas de aparcamiento en un cuerpo edificado perteneciente a tres firmas comerciales de Eschborn, entre Francfort y el monte Feldberg, en el Taunus, gracias a la construcción de una planta superior.

Todos estos edificios o cubiertas de aparcamiento se distinguen de los demás, ventajosamente, por sus amplias estructuras portantes de acero que permiten el fácil aparcamiento de los vehículos a pesar de los apoyos intermedios ofreciendo, además, una duradera protección anticorrosiva gracias al sistema de galvanizado al fuego.

La cubierta de aparcamiento de Eschborn se encuentra aproximadamente a 1,5 m por encima del nivel de la calle. Se accede fácilmente a la misma desde la calle de dirección única, a través de una rampa doble axil (sobre cuatro soportes HEA-IPBL 360).

Desde la rampa doble se llega - a causa de la curva de entrada, relativamente larga- por una ancha pista doble, axil, a los aparcamientos dispuestos a ambos lados, ya que se aparca en "ángulo recton. La cubierta inferior se encuentra aproximadamente a $1,5 \mathrm{~m}$ por debajo del nivel de la calle y es también fácilmente accesible desde la ya mencionada calle, de dirección única, a través de dos rampas laterales situadas más o menos en los cuartos externos de los lados cortos. Desde estas rampas se llega por dos pistas coaxiles y, por una curva de entrada corta, a cuatro filas de 10 plazas de aparcamiento, cada una, con un ángulo de aproximadamente $60^{\circ}$ con respecto al eje longitudinal (se aparca oblicuamente, en "ángulo torcido»). 


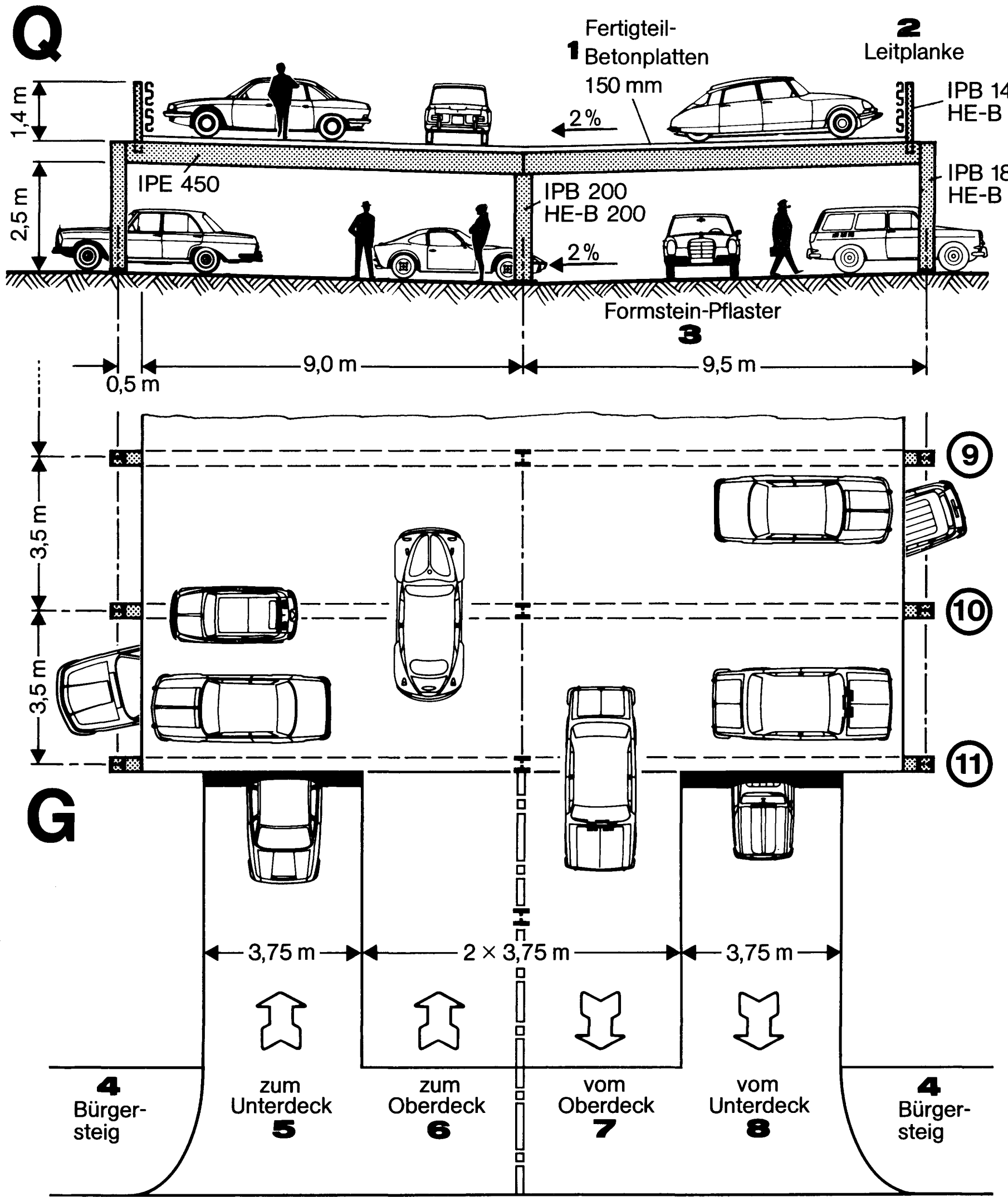

$\boldsymbol{9}$ Ginnheimer Straße 
A la cubierta inferior se entra y sale también bajo cubierta, a pesar de que los vehiculos de las filas de aparcamiento exteriores se encuentran ya con su parte delantera o posterior fuera de la cubierta superior. En total se dispone de $28+40=68$ plazas de aparcamiento para los clientes y empleados, en una superficie básica de aproximadamente 24 × 35 metros.

La estructura portante de acero se compone de once pilares, en once filas a $3,5 \mathrm{~m}$ de distancia respectivamente. Sobre los mismos se encuentran vigas continuas (IPE 450) de 9,5 m de longitud, dobladas ligeramente a fin de conseguir una inclinación del 2 por 100 para la evacuación de las aguas. Dichas vigas se estabilizan a la altura del cordón inferior, en el eje central, por medio de una barra longitudinal (perfil hueco rectangular de $50 \times 50 \times 5 \mathrm{~mm}$.) y sobre todo por medio de las placas de cubierta, de hormigón armado, de la planta superior. Se han empleado placas de hormigón armado prefabricadas de $3,5 \times 2,25 \times 0,15 \mathrm{~m}$, a las cuales se aplicó, después del relleno de las juntas, una fina capa de hormigón superior.

Por debajo de la planta superior sobresalen las citadas vigas $0,5 \mathrm{~m}$, y sobre ellas se atornillan, mediante placas frontales, unos postes bilaterales HEB 140 para dos bordillos-guia, como elementos de protección contra las caidas. Todos los puntos de introducción de fuerza precitados están provistos de tiras rayadas. Las uniones roscadas resistentes al galvanizado, al fuego, permiten también el galvanizado de los pasadores sin necesidad de desmontar o partirlos por doble inmersión oblicua en el baño de estaño.

\section{résumé}

COUVERTURE DEMONTABLE EN ACIER POUR UN PARKING A ESCH. BORN - REPUBLIQUE FEDERALE D'ALLEMAGNE

Klaus Idelberger, ingénieur

\section{summary}

REMOVABLE STEEL CAR PARK ROOF IN ESCHBORN - GERMAN FEDERAL REPUBLIC

Klaus Idelberger, Engineer

A two-level car park is described, made of a metal structure, with reinforced concrete cladding and units measuring $3.5 \times 2.25 \times$ $\times 0.15 \mathrm{~m}$.

The structure can be dismantled, with a view to transporting it and adding possible extensions, which constitutes a functional and different characteristic.

Currently there is space for 68 vehicles distributed over an area of $24 \times 35$ metres.

\section{zusammenfassung}

DECKE EINER DEMONTIERBAREN PARKANLAGE AUS STAHL FUR KRAFTFAHRZEUGE IN ESCHBORN BUNDESREPUBLIK DEUTSCHLAND

Klaus Idelberger, Ingenieur

Es wird eine zweistöckige Parkanlage, bestehend aus einem Metallgerüst mit Stahlbetonfachwerken aufgrund von Einheiten von $3,5 \times 2,25 \times 0,15$ mit Hinterzwickel beschrieben.

Die Konstruktion ist demontierbar im Hinblick auf Verlegungen oder mögliche Erweiterungen, was ein funktionelles und differenziertes Merkmal darstellt.

Gegenwärtig verfügt man über 68 auf einer Fläche von $24 \times 35$ Metern verteilte Plätze. 